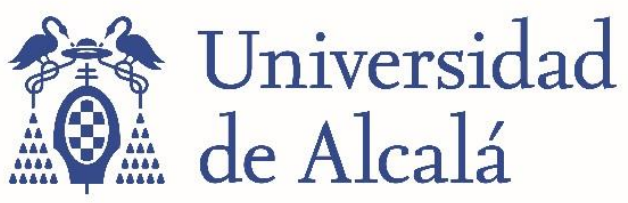

BIBLIOTECA

Document downloaded from the institutional repository of the University of Alcala: http://dspace.uah.es/dspace/

This is a postprint version of the following published document:

Rebollo et al., 2017. Spatial relationships and mechanisms of coexistence between dominant and subordinate top predators. Journal of Avian Biology, 48(9), pp.1226-1237.

Available at https://doi.org/10.1111/jav.01337

(C) 2017 Nordic Society Oikos

(C) 2017 Th e Authors. Journal of Avian Biology

(Article begins on next page)
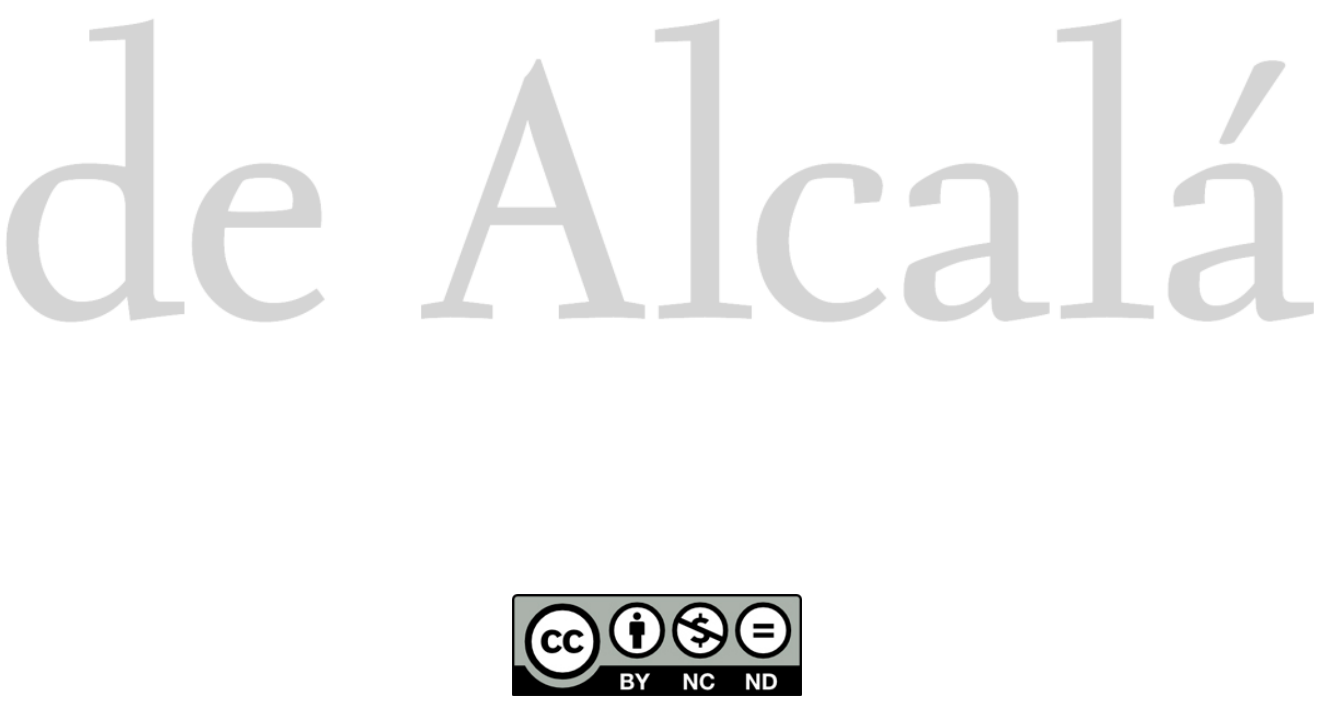

This work is licensed under a

Creative Commons Attribution-NonCommercial-NoDerivatives

4.0 International License. 


\title{
Spatial relationships and mechanisms of coexistence between dominant and subordinate top predators
}

\author{
S. Rebollo, S. Martínez-Hesterkamp, G. García-Salgado, L. Pérez-Camacho, J. M. Fernández-Pereira \\ and J. Jenness
}

S. Rebollo (http://orcid.org/0000-0001-8797-1122) (salvador.rebollo@uah.es), S.Martinez-Hesterkamp, G. García-Salgado, L. Pérez-Camacho and J. M. Fernández-Pereira, Forest Ecology and Restoration Group, Depto de Ciencias de la Vida, Univ. de Alcalá, Alcalá de Henares, Madrid, Spain. - J. Jenness, Flagstaff, AZ, USA.

\begin{abstract}
Most forest ecosystems contain a diverse community of top-level predators. How these predator species interact, and how their interactions influence their spatial distribution is still poorly understood.

Here we studied interactions among top predators in a guild of diurnal forest raptors in order to test the hypothesis that predation among competing predators (intraguild predation) significantly affects the spatial distribution of predator species, causing subordinate species to nest farther away from the dominant ones.

The study analyzed a guild in southwestern Europe comprising three raptor species. For 8 years we studied the spatial distribution of used nests, breeding phenology, intraguild predation, territory occupancy, and nest-builder species and subsequent nest-user species.

The subordinate species (sparrowhawk Accipiter nisus) nested farther away from the dominant species (goshawk $A$. gentilis), which preyed on sparrowhawks but not on buzzards Buteo buteo, and closer to buzzards, with which sparrowhawks do not share many common prey. This presumably reflects an effort to seek protection from goshawks. This potential positive effect of buzzards on sparrowhawks may be reciprocal, because buzzards benefit from old sparrowhawk nests, which buzzards used as a base for their nests, and from used sparrowhawk nests, from which buzzards stole prey. Buzzards occasionally occupied old goshawk nests.

These results support our initial hypothesis that interspecific interactions within the raptor guild influence the spatial distribution of predator species in forest ecosystems, with intraguild predation as a key driver. We discuss several mechanisms that may promote the coexistence of subordinate and dominant predators and the spatial assembly of this raptor guild: spatial refuges, different breeding phenology, spatial avoidance, low territory occupancy between neighboring nesting territories, nest concealment and protection, and diet segregation.
\end{abstract}

Most forest ecosystems contain a complex community of top-level predators differing in size, diet and hunting strategies (Reynolds and Meslow 1984). These predator species interact with one another through competition, predation (also designated as superpredation in this particular case, Lourenço et al. 2011), intraguild predation (a combination of predation and competition; Polis et al. 1989), and facilitation (where a species benefits another species without being negatively affected itself, Rebollo et al. 2011). These interactions can influence how the predator species are spatially distributed in the ecosystem (Kostrzewa 1991, Gamauf et al. 2013). This spatial distribution of predators can then determine the spatial distribution of predation pressures, especially when the predators are central-place foraging species and organize hunting activities from a central point, such as raptor nests during the breeding season (Byholm et al. 2012). If predators tend to nest far from one another, it may generate enemy-free areas for non-shared prey (Thomson et al. 2006, Mönkkönen et al. 2007). Conversely, if predators tend to nest close together, it may generate areas of more intense predation pressure on shared prey. Therefore, analyzing the spatial distribution of predators in the same guild has theoretical and applied (e.g. conservation strategies of communities of top predators) value in ecology because it provides insights into predator-prey systems and mechanisms of predator coexistence that are more realistic and integrated than insights gained from mono-specific approaches.

Several factors influence the spatial distribution of forestdwelling raptors. Cornulier and Bretagnolle (2006) classified them into first- and second-order factors, without implying an order of relative importance. First-order factors relate to the environmental heterogeneity characteristic of patchy environments, such as vegetation types, while second-order factors deal with social interactions between individuals of a population that lead to intraspecific association or repulsion. In the present study, we develop the concept of third-order factors, which refer to interspecific interactions between top 
predator species. Relatively few studies have attempted to disentangle the influence of third-order factors on the spatial distribution of top-level predators in forest ecosystems while taking into account first- and second-order factors (but see Katzner et al. 2003, Morosinotto et al. 2017). To a large extent, this gap in the literature reflects the difficulty of the task: for example, different first-, second- and thirdorder factors can produce similar patterns of proximity or distance between individuals at a given spatial scale, leading to misinterpretations about the causes of the observed spatial distribution (Cornulier and Bretagnolle 2006). In addition, the three levels of factors are not always independent. For example, habitat preference (first-order factors) may depend not only on population density (second-order) but also on interspecific interactions (third-order) such as intraguild predation (Newton 1986, Sergio et al. 2003).

In the present work we analyzed spatial relationships in a community of diurnal forest raptors comprised of three central-place foragers: northern goshawk Accipiter gentilis, common buzzard Buteo buteo and Eurasian sparrowhawk $A$. nisus, hereafter referred to as goshawk, buzzard and sparrowhawk. These three species often constitute the core of the diurnal forest-dwelling raptor guilds in Europe (Zuberogoitia and Martínez 2011). We studied breeding season relationships of the three species with the following specific objectives. First, to identify positive, negative and neutral relationships among species (third-order factors) by analyzing whether nests were located closer together or farther apart than predicted by chance, while taking into account intraspecific territoriality (second-order) and habitat preferences of each species (first-order). We hypothesized that interspecific interactions, particularly intraguild predation, would determine the spatial organization of the predator guild. We expected that the goshawk, as a potential predator of adults and nestlings of the other two species, would act as the dominant species (Kostrzewa 1991, Krüger 2002a,
Chakarov and Krüger 2010, Björklund et al. 2016). Buzzards and sparrowhawks, in turn, would act as subordinate species, so their nests should lie farther from used goshawk nests. We also expected that used buzzard and sparrowhawk nests would be located independently of each other, although they might lie close to each other in spatial refuges of goshawk predation.

Second, to identify possible explanations for non-random proximity/separation between nests of different species. These explanatory variables included: breeding phenology, the consecutive use of the same nest by different species, simultaneous occupancy between neighboring nesting territories of different species, and intraguild predation. Although some studies of interactions among these three species have been published (Fasola and Zanghellini 1993, Selås 1997, Hakkarainen et al. 2004), we are unaware of previous studies analyzing the implications of these interactions on the spatial distribution and assembly of this forest raptor guild (but see Chakarov and Krüger 2010, Björklund et al. 2016, Mueller et al. 2016).

\section{Material and methods}

\section{Study area}

The study was conducted on the peninsula of Morrazo $\left(183.3 \mathrm{~km}^{2}\right)$ in northwestern Spain (lat. $42^{\circ} 20^{\prime}$, long. $8^{\circ} 47^{\prime}$ ) (Fig. 1), an area of rough topography at a mean altitude of $213 \mathrm{~m}$ (range $0-646 \mathrm{~m}$ ). The climate is humid oceanic with an average annual precipitation of $1586 \mathrm{~mm}$ and temperature of $14.4^{\circ} \mathrm{C}$ (Carballeira et al. 1983). Nearly half $(42.5 \%)$ the area is covered by planted or regenerated eucalyptus forests Eucalyptus globulus (a perennial exotic species of Australian origin), English oaks Quercus robur and maritime pines Pinus pinaster; $8.4 \%$ is covered by young

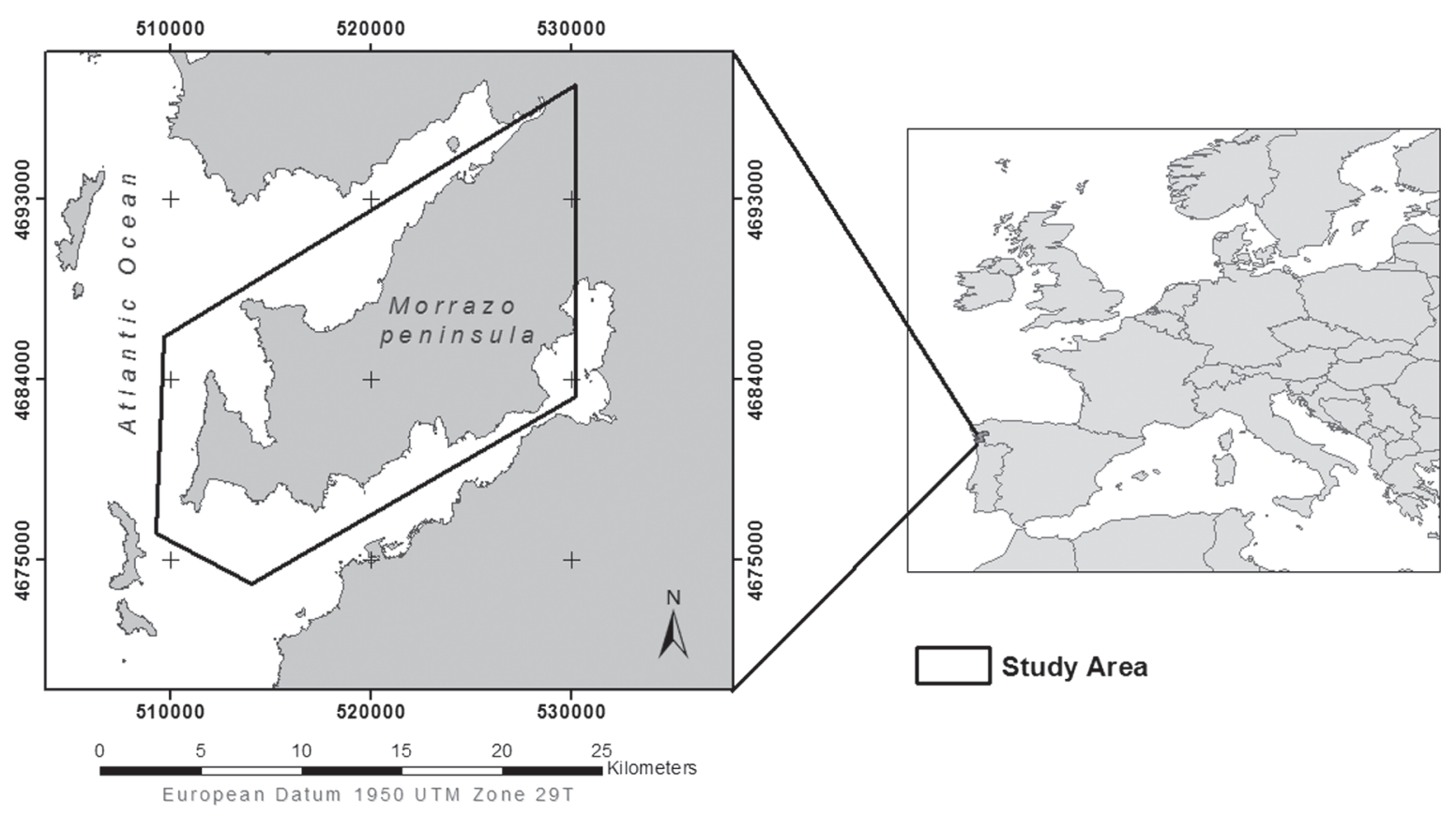

Figure 1. Location of the study area in the southwest of Europe and the northwest of the Iberian Peninsula. 
tree formations; $35.4 \%$, by crops and scattered houses; and $13.6 \%$, by urban areas, scrublands and beaches. Forests are mainly private with low-intensity harvests in small-sized plots (80\% smaller than 0.5 ha; Álvarez-Taboada 2005) with varied rotations; some sites have rotations exceeding $50 \mathrm{yr}$. From aerial photographs and detailed field surveys we generated a map of the different forest types (Table 1 and Supplementary material Appendix 1 Fig. A1). The study area has a relatively high breeding pair density of goshawk, buzzard and sparrowhawk (Rebollo et al. 2011, Pérez-Camacho et al. 2015). Other diurnal forest raptors such as European honey buzzard Pernis apivorus are rare in this area $(0-5$ breeding pairs $\mathrm{yr}^{-1}$ ) and were not considered in this study. Eagle owls Bubo bubo, which may prey on goshawk and buzzard, are not present in the study area. Tawny owl Strix aluco is present in the area and may prey on nestlings of sparrowhawk (Newton 1986).

\section{Characteristics of raptor species}

Goshawks and buzzards are medium-sized raptors and sparrowhawks are small raptors. Weights range from 814$1510 \mathrm{~g}$ (goshawk), 804-923 g (buzzard) and 151-268 g (sparrowhawk) (Ferguson-Lees and Christie 2001). The three species show intraspecific territoriality during the breeding season (Newton et al. 1977, Penteriani and Faivre 1997, Reich et al. 2004) and show differences in breeding phenology. Buzzards are usually the first to start breeding and sparrowhawks the last, starting more than a month after buzzards (Newton 1979, p. 97).

The three species are sympatric (Selås and Rafoss1999, Zuberogoitia and Martínez 2011) and are frequently found nesting in the same forest patch during the same breeding season or over multiple years (Solonen 1993). The three species show similar nesting requirements, however, some species differences with regard to topography and forest structure have also been reported. For example, Fasola and Zanghellini (1993) reported elevational differences among these species, and Newton (1986) reported that sparrowhawks use younger forest stands than goshawks. Breeding pairs of goshawks and buzzards usually maintain several nests, which can be used in alternating years by the same or different species (Krüger 2002a). Sparrowhawks, in contrast, usually build a new nest near the previous year's nest (Newton 1986). The three species probably compete for the best nesting sites, being the goshawk the dominant species that displaces the other two from the nesting sites and even occupies their nests (Newton 1986, Kostrzewa 1991, Krüger 2002b, Kenward 2006). In addition, they might compete for certain hunting habitats; sparrowhawks with buzzards, as they both use young forests patches and clearings, and goshawks with sparrowhawks, when hunting in old woodland (Selås and Rafoss 1999). The three species can also compete for food as they show some overlap in diet, with sparrowhawks and buzzards showing the less overlap (Tubbs 1974, Newton 1986, Cramp and Simmons 2004, Kenward 2006). Goshawks feed on small-sized birds with this component of the diet varying locally (Rutz et al. 2006), even though the main components of their diet are usually medium-sized birds and mammals, including raptors such as sparrowhawks and, especially in northern latitudes, buzzards (Kostrzewa 1991, Krüger 2002a, García-Salgado et al. 2015). Thus, goshawks are super-predators (Lourenço et al. 2011) that may influence nest spacing of other forestdwelling raptors (Gamauf et al. 2013). Buzzards feed mainly on small- and medium-sized mammals and reptiles, occasionally preying on birds and smaller raptors such as sparrowhawks (Sergio et al. 2002, Zuberogoitia et al. 2006, Tapia et al. 2007). Sparrowhawks are primarily bird-eating raptors, feeding on small- and medium-sized birds (Mañosa and Oro 1991, Selås 1993). Outside the breeding season sparrowhawk's diet probably overlaps more with goshawk's diet as female sparrowhawks hunt larger prey than male sparrowhawks do (Newton 1986).

\section{Interspecific distances and null models}

The study area was systematically surveyed for goshawks and buzzards for eight years (2004-2011) and for sparrowhawks for six years (2006-2011) in order to locate as many used nests as possible. The relatively small size of the study area and the territoriality, site fidelity and reuse of the nests by the species facilitated detection of used nests. We considered used nests to be those where incubation was observed (Reich et al. 2004). Each year, the nearest-neighbor distance (NND) between used nests of the same species and the nearestneighbor distance between used nests for each pair of species were measured. For each pair of species, we estimated the shortest distance between used nests in two, non-equivalent 'directions': from species A to species B, and from species B to species A (Fig. 2). This is because calculating the shortest distance from $A$ to $B$ takes into account all used nests of species A but only the closest used ones of species B, while the opposite is true for calculating the shortest distance from $\mathrm{B}$ to A. Taking as an example the sparrowhawk and goshawk, the estimation of the distances $\mathrm{A}$ to $\mathrm{B}$ and $\mathrm{B}$ to $\mathrm{A}$ allows us to check if the effect of goshawk was on all sparrowhawk nests or only on the subgroup of sparrowhawk nests located closer to goshawk nests.

To determine whether species were nesting closer or farther apart from each other than one would expect by chance, we measured the distance between each used nest of a species and the nearest simulated 'nest' of each of the other two species located at random positions in the forest habitat generated using a null spatial model. For each year and species, 100 simulated sets of randomly distributed nests were used, with each set featuring a number of random nests equal to the number of used nests for that particular species in that particular year (Table 2). For example, in 2007, goshawks had 18 used nests in the study area, so we generated 100 simulated sets of 18 randomly distributed nests. This allowed us to calculate, for each used nest and year, the distribution of interspecific distances corresponding to the null hypothesis, which stipulated that interspecific nest distances purely reflected chance. The null model took into account habitat preferences (first-order factor) and intraspecific territorial behavior (second-order factor) of each species. That is, we distributed simulated 'nests' at random on maps which included all forest types, but excluded forest patches smaller than the smallest patch used by a given species and elevations above the maximum elevation where nests were found. In addition, the simulated 'nests' were 


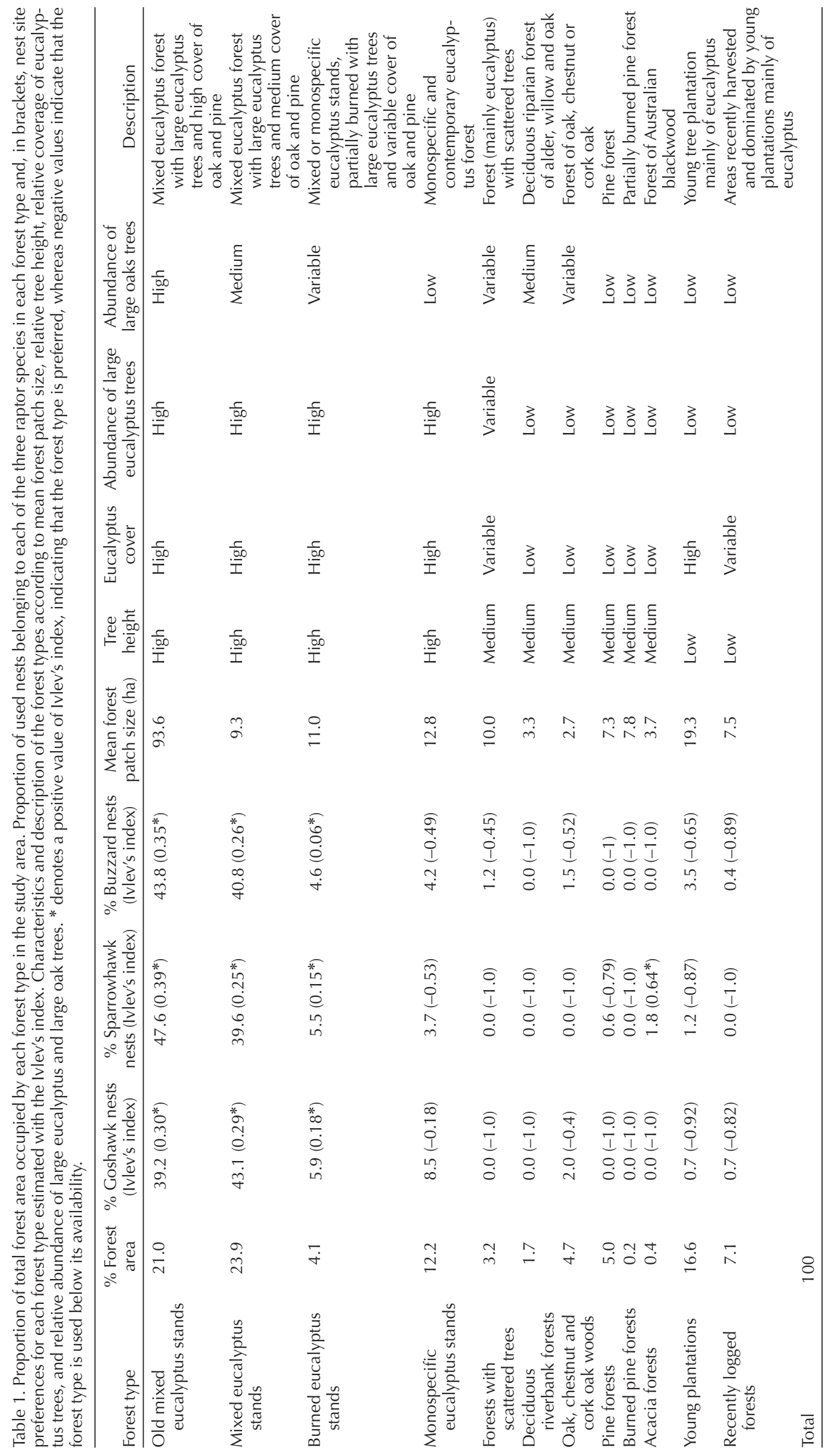




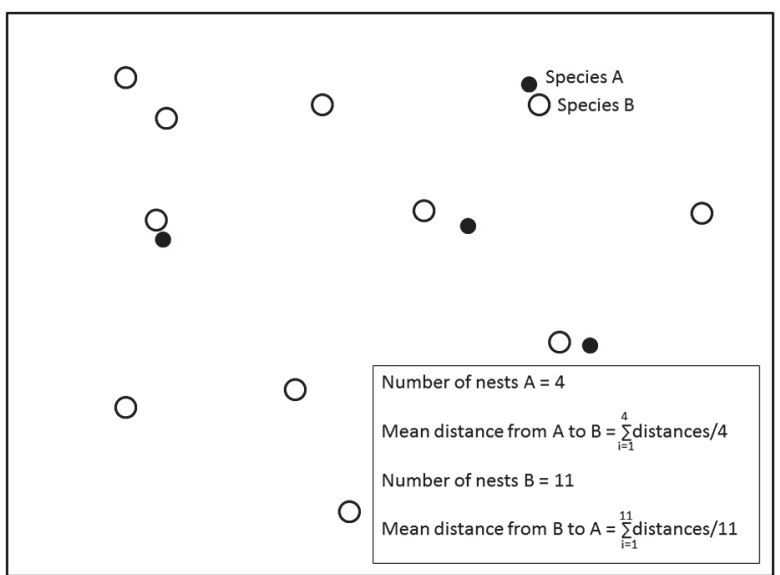

Figure 2. The interspecific nearest-neighbor distances (NNDs) from used nests of the species A to species B, and from species B to species $A$ are not equivalent. Note that, in this example, the NNDs from species A to species B are much shorter than the NNDs from species $B$ to species $A$, and for the calculations of the NNDs from species A to species B, only the four closest used nest of species B were taken into account.

distributed in each forest type with the same proportion as the proportion of observed nests in that forest type (Table 1). And finally, they were constrained to respect observed intraspecific distances; the minimum distance between simulated 'nests' was always equal or larger than the minimum intraspecific distance between nests for that species in that particular year. Therefore, the null model assumed that the forest cover of the study area was heterogeneous and that not all forest environments were equally likely to have used nests. A Geographic Information System (ArcGIS 10.0, ESRI 2010) was used to build these maps of forest environments, generate the randomly distributed simulated nests and calculate the aforementioned distances.

We used two approaches to compare observed and simulated distances. In the first approach, we used generalized linear mixed models (GLMM) to detect whether the average distances between nests for each pair of species were larger or smaller than simulated distances, in order to detect whether nests of different species were closer together or farther apart than predicted by chance. For each pair of species we fitted a GLMM using the NND as response variable and the type of distance (observed or simulated) as the fixed factor. Year, territory and nest were included as random factors (full model), which allowed to control for the spatiotemporal dependence of the data. Each of the above models was compared with its null model, which did not include the fixed factor. We assessed model fit using the Akaike information criterion modified for small sample sizes (AICc). If the AICc of the full model was smaller than that of the null model by more than 6 points (Symonds and Moussalli 2011), we concluded that the full model was more plausible than the null model; in other words, the observed distances were different from those expected by chance. GLMMs were calculated using the 'Ime4' package (Bates et al. 2013) in R 3.0.2 (R Development Core Team).

Table 2. Mean intraspecific and interspecific nearest-neighbor distances (NNDs) of northern goshawk, Eurasian sparrowhawk and common buzzard throughout the study period (2004-2011). Each year, the NND between used nests of the same species and the NND between used nests for each pair of species were measured. The table also shows the NNDs between the nesting territories that were active for at least one year during the study period.

\begin{tabular}{|c|c|c|c|c|c|c|}
\hline \multirow[b]{2}{*}{ Species } & \multirow[b]{2}{*}{ Year/territories } & \multirow[b]{2}{*}{$\mathrm{n}$} & \multirow[b]{2}{*}{ Mean intraspecific NND \pm SE $(m)$} & \multicolumn{3}{|c|}{ Mean interspecific NND \pm SE $(\mathrm{m})$} \\
\hline & & & & To goshawks & To sparrowhawks & To buzzards \\
\hline \multirow[t]{9}{*}{ Goshawk } & 2004 & 22 & $2170.8 \pm 136.2$ & & ND & $1101.6 \pm 137.8$ \\
\hline & 2005 & 20 & $2305.3 \pm 153.8$ & & ND & $1118.2 \pm 115.6$ \\
\hline & 2006 & 20 & $2198.9 \pm 122.8$ & & $1840.4 \pm 242.0$ & $992.3 \pm 136.3$ \\
\hline & 2007 & 18 & $2209.0 \pm 212.3$ & & $1464.2 \pm 228.4$ & $1057.7 \pm 211.7$ \\
\hline & 2008 & 18 & $2404.3 \pm 179.8$ & & $1018.4 \pm 105.5$ & $979.8 \pm 168.5$ \\
\hline & 2009 & 18 & $2070.5 \pm 132.8$ & & $1297.7 \pm 147.7$ & $1102.7 \pm 212$ \\
\hline & 2010 & 18 & $2433.8 \pm 216.8$ & & $1272.0 \pm 183.3$ & $860.5 \pm 118.7$ \\
\hline & 2011 & 19 & $2080.2 \pm 142.1$ & & $1567.4 \pm 270.3$ & $1193.5 \pm 208.0$ \\
\hline & Territories & 29 & $1933.3 \pm 84.4$ & & $681.8 \pm 83.8$ & $397.2 \pm 86.7$ \\
\hline \multirow[t]{7}{*}{ Sparrowhawk } & 2006 & 16 & $1774.3 \pm 268.0$ & $1253.6 \pm 139.5$ & & $874.1 \pm 180.4$ \\
\hline & 2007 & 22 & $1718.8 \pm 182.8$ & $1359.1 \pm 153.3$ & & $813.3 \pm 175.3$ \\
\hline & 2008 & 37 & $1310.3 \pm 84.7$ & $1341.4 \pm 100$ & & $731.6 \pm 103.2$ \\
\hline & 2009 & 26 & $1486.2 \pm 140.4$ & $1687.4 \pm 222.6$ & & $830.5 \pm 150.7$ \\
\hline & 2010 & 25 & $1592.4 \pm 105.8$ & $1330.4 \pm 125.7$ & & $773.5 \pm 112.1$ \\
\hline & 2011 & 24 & $1525.1 \pm 136.5$ & $1419.4 \pm 179$ & & $1022.1 \pm 105.1$ \\
\hline & Territories & 57 & $1052.5 \pm 55.9$ & $878 \pm 57.0$ & & $385 \pm 49.5$ \\
\hline \multirow[t]{9}{*}{ Buzzard } & 2004 & 29 & $1259.0 \pm 170.3$ & $1036.6 \pm 110.5$ & ND & \\
\hline & 2005 & 26 & $1591.3 \pm 167.1$ & $1143.1 \pm 116.0$ & ND & \\
\hline & 2006 & 28 & $1657.4 \pm 169.6$ & $1174.5 \pm 125.9$ & $1531.3 \pm 235.9$ & \\
\hline & 2007 & 39 & $1082.8 \pm 102.1$ & $1288.2 \pm 134.0$ & $1086.8 \pm 124.9$ & \\
\hline & 2008 & 32 & $1494.9 \pm 166.1$ & $1161.8 \pm 122.1$ & $608.6 \pm 90.3$ & \\
\hline & 2009 & 34 & $1295.4 \pm 121.8$ & $1931.5 \pm 269.0$ & $934.7 \pm 123.4$ & \\
\hline & 2010 & 42 & $1137.1 \pm 86.8$ & $1296 \pm 100.5$ & $1157.3 \pm 116.7$ & \\
\hline & 2011 & 30 & $1071.5 \pm 125.8$ & $1209.3 \pm 118.6$ & $1450.6 \pm 170.3$ & \\
\hline & Territories & 84 & $742.3 \pm 38.8$ & $762.2 \pm 58.7$ & $554.9 \pm 54.3$ & \\
\hline
\end{tabular}

Note: ND, not determined. 
In the second approach we visually compared the frequencies of observed and simulated inter-nest distances to identify potential tolerance buffers among species. We show frequency bar graphs of observed interspecific distances and of randomly distributed simulated interspecific distances for each pair of species. Our goal was to detect ranges (bins) of observed interspecific distances that were substantially smaller or larger than the corresponding distances expected by chance. Frequencies of observed and simulated distances were estimated separately for each year, and the mean frequency and SE were calculated over the entire study period.

To better consider the full dimensionality of the interspecific nesting landscape and to explore the potential influence of second or third interspecific neighbor nests located further than the nearest neighbor nest, we applied the same analyses performed for the NNDs to the second and third nearest neighbors. However, since we did not find any significant effect of second and third neighbors (Supplementary material Appendix 1 Table A1 and A2), we only show and discuss results of the NNDs.

We estimated the preference of each species for different forest types using the Ivlev index:

$S=\frac{(r-p)}{(r+p)}$

where $r$ represents the percentage of nests found in a forest type and $p$ represents the availability of that forest type (percentage of total forest area occupied by that forest). This index ranges from -1 to +1 , and positive values were considered to indicate preference for a given forest type.

\section{Breeding phenology, territory occupancy between interspecific neighboring nesting territories, nest builders and users, and intraguild predation}

To understand the spatial relationships among the species we summarized breeding phenology for the three species in the study area because only the species that breeds late in the breeding season have the real option to distance or to approach the other species. The simultaneous occupancy between neighboring nesting territories of different species can also help to understand the spatial relationships among the species. For example, if goshawks predate on neighboring sparrowhawks and sparrowhawks nest later than goshawks, we would expect nesting goshawk territories that neighbor occupied sparrowhawk territories to be more frequently occupied than those goshawk territories located farther away from occupied sparrowhawk territories. Similarly, if sparrowhawks benefit from neighboring buzzards, and sparrowhawks nest later than buzzards, we would expect nesting buzzard territories that neighbor occupied sparrowhawk territories to be more frequently occupied than those buzzard territories located farther away from occupied sparrowhawk territories. Thus, for each used nest of buzzard and sparrowhawk, we analyzed whether the neighboring territories of the other two species were occupied or unoccupied in the same breeding season. We considered occupied territories to be those containing a used nest (incubation observed) in a particular breeding season. To establish the neighboring territories, we took into account the mean distance between pairs of species
(Table 2). For the pairs of species with relative short mean distance (sparrowhawk-buzzard and buzzard-goshawk) we considered neighboring territories to be those whose nest (or the centroid of the alternative nests when the territory had more than one nest) was located less than $400 \mathrm{~m}$ from an used nest of the other species. For the pair of species with a relative high mean distance (sparrowhawk-goshawk) we increased the threshold distance to $600 \mathrm{~m}$. In the three cases, the threshold distance was less than half the mean distance. Using a chi-squared test, we compared the frequency of occupancy in neighboring territories with the frequency of occupancy across all nesting territories of that species. These statistical analyses were performed using Statistica 8.0 (StatSoft 2007), using a significance level of $\alpha=0.05$.

New nests were catalogued each year, allowing us to track nest-builder and nest-user species and to determine if the species that used the nest is the same that built it, and how frequently this happens. Intraguild predation by goshawk was studied by collecting prey remains and installing digital photo cameras in 80 goshawk nests (García-Salgado et al. 2015). In addition, cameras were placed in 19 sparrowhawk nests (recording a total of $19427 \mathrm{~h}$ during the period of nestlings and fledglings) to estimate the rate of predation of sparrowhawk nestlings.

\section{Results}

\section{Density of nests and nesting territories, intraspecific distances, and habitat preferences}

The average number of used nests per year $( \pm$ SE) during the study period was $32.5 \pm 2.0$ for buzzards, corresponding to a density of $17.7 \pm 1.1$ breeding pairs $100 \mathrm{~km}^{-2} ; 25.0 \pm 2.8$ for sparrowhawks, or $13.6 \pm 1.5$ breeding pairs $100 \mathrm{~km}^{-2}$; and $19.1 \pm 0.5$ for goshawks, or $10.4 \pm 0.3$ breeding pairs $100 \mathrm{~km}^{-2}$ (Table 2). The number of used goshawk nests each year decreased during the study period ( $\mathrm{n}=8 \mathrm{yr}$, Pearson's $r=-0.74, p=0.036$ ), whereas the numbers of used buzzard and sparrowhawk nests showed no trends over time. The number of different nesting territories that were occupied during at least one year of the 8-yr study period for goshawks and buzzards or during the 6-yr study period for sparrowhawks was 29 for goshawks, 84 for buzzards, and 57 for sparrowhawks. The mean intraspecific nearest-neighbor distances (NNDs) are shown in Table 2. Goshawks showed the longest mean intraspecific NNDs between used nests $(\mathrm{n}=8$, mean \pm SE, $2234.1 \pm 162.1 \mathrm{~m})$, followed by sparrowhawks $(\mathrm{n}=6,1567.8 \pm 153 \mathrm{~m})$ and buzzards $(\mathrm{n}=8$, $1323.7 \pm 138.7 \mathrm{~m}$ ).

The three species preferred to nest in similar forest types (Table 1) and had positive values of the Ivlev's index for 'old mixed eucalyptus stands', 'mixed eucalyptus stands', and 'burned eucalyptus stands', suggesting that these forest types were used above their availability by the three species. These types correspond to the stands with oldest and tallest eucalyptus trees and with the most diverse tree composition. These three types of eucalyptus stands occupied $49 \%$ of the forest area and contained $88 \%$ of goshawk nests, $89 \%$ of buzzard nests and $93 \%$ of sparrowhawk nests. The sparrowhawk also showed positive values of the 
Ivlev's index for stands of Australian blackwood Acacia melanoxylon, but the availability of this forest type and the number of sparrowhawk nests involved was too low as to make inferences from this result (Table 1). Goshawks and sparrowhawks always bred in forest patches larger than 4.1 ha; buzzards, in patches larger than 6.0 ha. Regarding to elevation, goshawks nested below $340 \mathrm{~m}$; sparrowhawks and buzzards, below $370 \mathrm{~m}$.

\section{Interspecific nearest-neighbor distances (NNDs)}

The longest mean interspecific NNDs between used nests were found from goshawk to sparrowhawk $(\mathrm{n}=6,1410 \pm 115.1 \mathrm{~m}$, Table 2), followed by sparrowhawks to goshawk $(\mathrm{n}=6$, $1398.6 \pm 61.7 \mathrm{~m})$, buzzard to goshawk $(\mathrm{n}=8,1280.1 \pm 97.5$ $\mathrm{m})$, buzzard to sparrowhawk $(\mathrm{n}=6,1128.2 \pm 138.6 \mathrm{~m})$, goshawk to buzzard $(\mathrm{n}=8,1050.8 \pm 41.3 \mathrm{~m})$, and sparrowhawk to buzzard $(\mathrm{n}=6,840.9 \pm 50.1 \mathrm{~m})$.

Observed distances between goshawk and buzzard used nests were similar to simulated distances expected by chance (Table 3). Observed sparrowhawk-buzzard distances were smaller than simulated ones, whereas buzzard-sparrowhawk distances were similar to simulated ones (Table 3 ). This indicates that the closer-than-random proximity of sparrowhawk nests to buzzard nests was detectable only when considering the subgroup of buzzard nests closest to sparrowhawk nests. An average of $33.5 \%$ of the sparrowhawk nests were located within $400 \mathrm{~m}$ from used buzzard nests across the study period, compared to only $18 \%$ by chance (Fig. 3).

Observed distances between goshawk-sparrowhawk used nests were greater than simulated ones, while sparrowhawkgoshawk distances were similar to simulated ones (Table 3). This indicates that the larger-than-random separation between sparrowhawk and goshawk nests was detectable only when considering the subgroup of sparrowhawk nests closest to goshawk nests. Used sparrowhawk nests were found less frequently than expected by chance at distances less than $600 \mathrm{~m}$ from used goshawk nests (Fig. 3), but more frequently than chance at distances of 1000-1400 m.

\section{Territory occupancy between interspecific neighboring nesting territories}

Over the entire study period, goshawk territories (a total of 29 territories, Table 2) were occupied on average $66 \%$ of the time, significantly more than the average of $30 \%$ for the subgroup of goshawk territories within $600 \mathrm{~m}$ of used sparrowhawk nests (chi-squared $=23.10, \mathrm{df}=1$, $\mathrm{p}<0.001$ ), and significantly more than the average of $45 \%$ for the subgroup of goshawk territories within $400 \mathrm{~m}$ of used buzzard nests (chi-squared $=16.55, \mathrm{df}=1, \mathrm{p}<0.001$ ). Of the 14 occasions that we observed buzzards using nests built originally by goshawks, the goshawks were not nesting in that same territory on 11 occasions.

Over the entire study period all buzzard territories (a total of 84 territories, Table 2) were occupied on average $39 \%$ of the time, significantly less often than the $53 \%$ for the subgroup of buzzard territories within $400 \mathrm{~m}$ of used sparrowhawk nests (chi-squared $=7.32, \mathrm{df}=1, \mathrm{p}<0.007)$.

\section{Breeding phenology, construction and use of nests}

The three species showed differences in breeding phenology. The egg-laying period began approximately $12 \mathrm{~d}$ earlier for buzzards (average laying date $=30 \mathrm{March}$, range $=26$ February to 23 April) than for goshawks (average laying date $=11$ April, range $=17$ March to 19 May), and approximately one month and a half earlier for goshawks than for sparrowhawks (average laying date $=24$ May, range $=22$ April to 29 June). Goshawks built 45 new nests, of which nine $(20 \%)$ were subsequently used by buzzards. Buzzard built 56 new nests, none of which were used later by goshawks. Sparrowhawks built 183 new nests and reused fewer than $6 \%$ of them in the following year. Four $(2.2 \%)$ of the sparrowhawk nests were subsequently used by buzzards and one $(0.5 \%)$ by goshawks. During the study period, buzzards used 120 different nests and goshawks 70 , meaning that $3.3 \%$ of nests used by buzzards and $1.4 \%$ used by goshawks were enlargements of nests originally built by sparrowhawks. Sparrowhawks did not use nests built by the other two species.

\section{Intraguild predation by goshawks of sparrowhawks and buzzards}

Goshawks captured 7 sparrowhawks (less than $0.3 \%$ of prey items identified; $n=2618$ prey items, García-Salgado et al. 2015), of which 1 was a nestling, 1 was a fledgling and 5 were adults. No goshawk prey items were identified as being buzzards. In one of 19 sparrowhawk nests (5.3\%) where we installed cameras, predation of sparrowhawk nestlings by

Table 3. Results of generalized linear mixed models (GLMM) used to compare observed nearest-neighbor distances (NNDs) between used nests with simulated distances expected by chance for each pair of raptor species (northern goshawk, Eurasian sparrowhawk and common buzzard). The full model was considered more plausible than the null model when the AICC of the full model (AICC $)_{1}$ was lower than that of the null model $\left(\mathrm{AICc}_{0}\right)$ by more than 6 points $\left(\Delta_{\mathrm{i}}>6\right)$. Coefficients of the full model denote mean distances predicted by the model.

\begin{tabular}{lccrrr}
\hline Interspecific nest comparison & $\mathrm{AlCC}_{0}$ & $\mathrm{AlCC}_{1}$ & $\Delta_{\mathrm{i}}$ & $\alpha \pm \mathrm{SE}$ & $\beta \pm \mathrm{SE}$ \\
\hline Goshawk-sparrowhawk & 178909 & 178900 & 9 & $1306.40 \pm 72.74$ & $222.14 \pm 78.83$ \\
Sparrowhawk-goshawk & 244178 & 244180 & -2 & $1381.58 \pm 30.64$ & $49.52 \pm 65.00$ \\
Goshawk-buzzard & 242187 & 242189 & -2 & $1120.59 \pm 45.30$ & $2.90 \pm 49.19$ \\
Buzzard-goshawk & 418323 & 418325 & -2 & $1303.45 \pm 22.10$ & $2.15 \pm 43.93$ \\
Sparrowhawk-buzzard & 237493 & 237478 & 15 & $1107.54 \pm 25.42$ & $-201.93 \pm 38.66$ \\
Buzzard-sparrowhawk & 328524 & 328525 & -1 & $1285.47 \pm 46.63$ & $-50.21 \pm 42.56$ \\
\hline
\end{tabular}

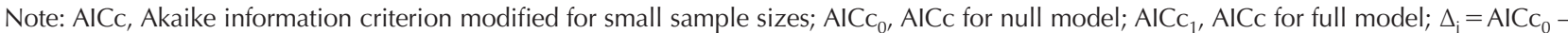
$\mathrm{AICC}_{1} ;=$ mean of random distances predicted by the full model; = mean difference between observed and random distances predicted by the full model. 
(a)

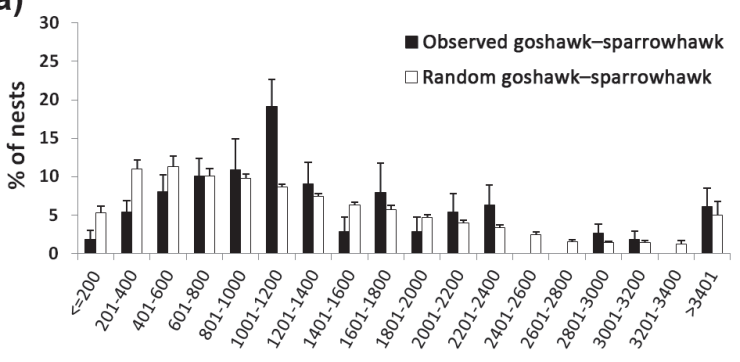

(c)

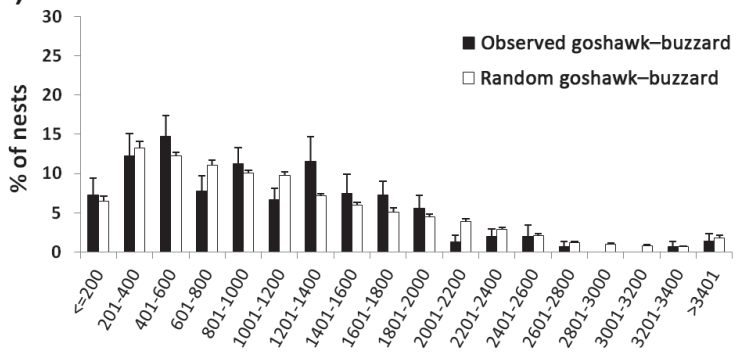

(e)

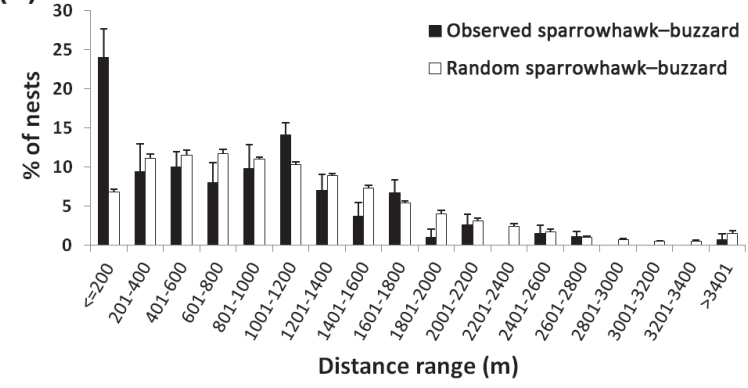

(b)

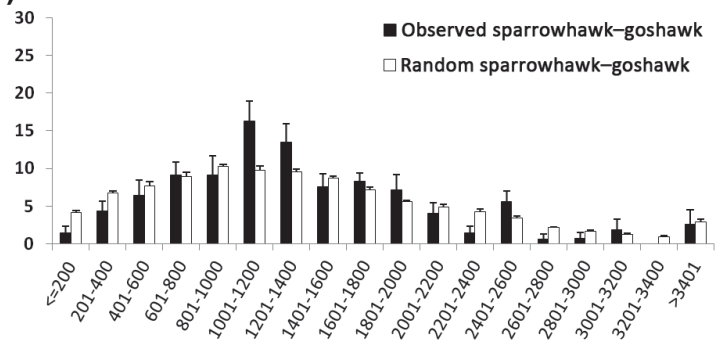

(d)

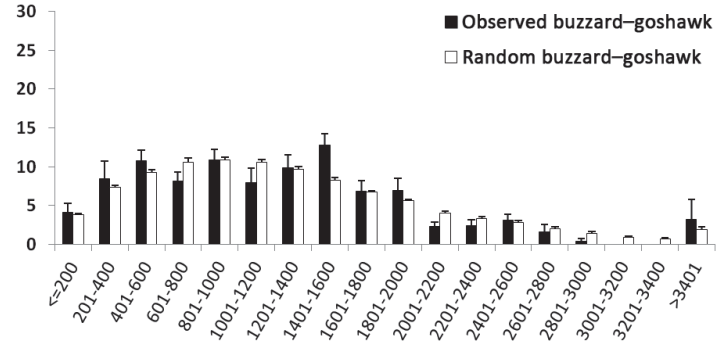

(f)

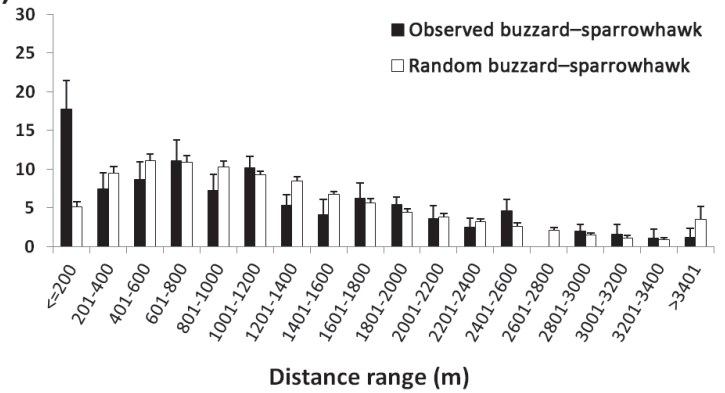

Figure 3. Frequency distribution of interspecific used nest distances, both observed (black bars) and expected by chance based on the null model (white bars), for the following species pairs: (a) goshawk-sparrowhawk, (b) sparrowhawk-goshawk, (c) goshawk-buzzard, (d) buzzard-goshawk, (e) sparrowhawk-buzzard, and (f) buzzard-sparrowhawk. The bar graphs show the mean percentage of nests located within each distance range (bin) for all years under study. Error bars indicate standard error.

goshawks was recorded. A buzzard was also detected in one sparrowhawk nest, but it was observed to steal only a prey item (common blackbird Turdus merula), without attacking the sparrowhawk nestlings.

\section{Discussion}

\section{Nest spacing in the raptor guild}

Interspecific interactions played an important role in the spatial organization of this diurnal forest raptor guild during the breeding season. Contrary to our initial hypothesis, our results suggest that goshawks were either not dominant over buzzards in our study area or they exerted negligible dominance. This may explain why we did not observe a) predation by goshawks of buzzard nestlings or adults (GarcíaSalgado et al. 2015), b) goshawks using nests built by buzzards, and c) buzzard nests showing larger-than-random separation from used goshawk nests during the study period. This lack of goshawk dominance contrasts with the situation in more northern European latitudes, where goshawks are larger than buzzards and often prey on buzzard nestlings and adults and occupy their nests (Kostrzewa 1991, Krüger 2002b, Hakkarainen et al. 2004, Chakarov and Krüger 2010, Björklund et al. 2016, Mueller et al. 2016). In more southern latitudes, such as our study area, goshawks and buzzards have more similar body sizes (Cramp and Simmons 2004, Kenward 2006), which may reduce predation on buzzards. However, our results do not exclude other ways of dominance of goshawk on buzzard because dominance is not only expressed by intraguild predation or a clear spatial avoidance. For example, there can be interference competition over nesting sites, with goshawks being capable of reducing buzzards breeding success (Krüger 2002a), and over hunting sites, altering the hunting behavior of buzzards (time and habitat), with no strong influence on nest site location.

Buzzards benefited from goshawk nest-building. Buzzards used $20 \%$ of nests built by goshawks, but this happened more often when goshawks were absent from the same nesting territory. The number of active goshawk breeding pairs decreased over the study period, which likely reflects in part illegal hunting activity, given that we observed traps for 
goshawk in the study area. This decline in goshawk breeding pairs releases abandoned goshawk nests that can be used by buzzards. This suggests that the interactions we observed between goshawks and buzzards may have been influenced at least to some extent by human activity. Therefore, further study is needed to examine these relationships in an environment with less human influence and to analyze other possibilities of interference competition of goshawk over buzzard (e.g. breeding performance, hunting behavior, etc).

In contrast to the weak spatial relationship between goshawk and buzzards nests, we found a stronger association between sparrowhawk and buzzard nests. Our initial hypothesis was that nests of both species would either show independent spatial distributions or would lie closer to each other than to goshawk nests in the event that the two species relied on the same refuge to avoid predation by goshawks. While we found that the two species nested closer together than would be expected by chance, it is unlikely that buzzards built nests closer to sparrowhawk because most buzzards begin laying eggs two months prior to sparrowhawks in our study area, when sparrowhawks have not yet built nests. It is also unlikely that our results reflect the use of old sparrowhawk nests by buzzards, given that only $3 \%$ of nests used by buzzards were enlargements of sparrowhawk nests. Instead we suggest sparrowhawks built nests closer to used buzzard nests in an effort to gain protection from goshawk. Support for this hypothesis was that the frequency of occupancy of buzzard territories neighboring occupied sparrowhawk territories was higher than the frequency of occupancy of buzzards across all nesting territories. Territory overlap should not promote strong competition between sparrowhawks and buzzards because the two species show little overlap in diet (Del Hoyo et al. 1994). Territory overlap does not pose high risks of predation on sparrowhawks because the predation rate of buzzards on sparrowhawk nestlings is low (Sergio et al. 2002, Zuberogoitia et al. 2006) and no case of predation was detected in our study. We suggest that this relationship is positive, since buzzards may indirectly protect sparrowhawks from predation by goshawks as our findings suggest that no buzzard adult, fledgling or nestling was predated by goshawk during our 8-yr study. Despite buzzards did not avoid goshawks, we think that this ability of buzzards to protect themselves from goshawks can indirectly benefit the sparrowhawks that nest near active buzzard nests. The positive relationship may even be mutual because buzzards may benefit from using old sparrowhawk nests as platforms to build their own nests, and from occasionally stealing prey from sparrowhawk nests. The spatial relationship between sparrowhawks and buzzards might also be because they share the same enemy-free space. However, we think that our results do not support that explanation because distances between goshawk and buzzard nests were similar to those predicted by a random distribution. At the same time, we caution that we are unable to exclude the possibility that buzzards and sparrowhawks nest close to each other because they share habitat preferences related to factors that we did not examine (e.g. forest edge). Future work should explore these possibilities.

Goshawk and sparrowhawk nest spacing suggest spatial avoidance by sparrowhawks. Goshawks preyed on sparrowhawk nestlings, fledglings and adults, and they were the only predator of sparrowhawk nestlings that we detected using nest cameras. Despite this intraguild predation and the high goshawk density (15.8 nesting territories $100 \mathrm{~km}^{-2}$ ), sparrowhawks managed to maintain a relatively high density of pairs (31.1 nesting territories $100 \mathrm{~km}^{-2}$ ).

\section{Mechanisms promoting coexistence between dominant and subordinate raptors}

Our findings support several mechanisms that may have promoted the coexistence of the subordinate predator and the dominant predator in our study area, and may provide keys for understanding the spatial structure (assembly) of this raptor guild.

1) Intense intraspecific competition among individuals of the dominant predator. This intense intraspecific competition among goshawk individuals favors the emergence of spatial refuges for subordinate species like sparrowhawks. Consistent with this idea, we observed that the mean intraspecific distance between goshawk pairs was much larger than the mean distance between sparrowhawks and goshawks (Table 2). This probably allowed sparrowhawks to find spatial refuges sufficiently far away from goshawk nests with concomitantly lower perceived risk of predation (see also Sergio et al. 2004). The relatively stable spatial distribution of goshawk territories over the study period probably helped subordinate predators like sparrowhawks to find these refuges more easily.

2) Late breeding phenology of the subordinate raptor and spatial avoidance from dominant raptor nests. Demonstrated increased nest-spacing of sparrowhawk from goshawks, the fact that $70 \%$ of sparrowhawks taken by goshawks were adults, and they started breeding at least one month later than goshawks in our study area, suggests sparrowhawks assess the risk of predation according to the distance from used goshawk nests and act accordingly. Such adaptive distancing from used goshawk nests has already been demonstrated in prey even smaller than sparrowhawk (Mönkkönen et al. 2007).

3) Low territory occupancy between neighboring nesting territories of the subordinate and dominant raptors. The fact that goshawk territories were occupied on average 66\% of the time and the subgroup of goshawk territories within $600 \mathrm{~m}$ of used sparrowhawk nests were only occupied an average of $30 \%$, suggests that sparrowhawks tended to locate their nests in areas with less active goshawk territories or in territories previously abandoned by goshawks. This likely facilitated the coexistence of the subordinate predator. Our results also supported that buzzards might use this mechanism to reduce interactions with goshawks.

4) Nest concealment and protection. Sparrowhawks also rely on nest concealment in complex woody structures in part to reduce risk of predation. Indeed, sparrowhawks placed a high percentage of nests $(93 \%)$ in the most structurally complex forests (mixed eucalyptus stands with high vertical development), even though these forests accounted for only $49 \%$ of the available area. Eucalyptus stands (an exotic tree in the study area), with their low rate of rotation, tall height and structural complexity, may favor the availability of nesting sites and predator coexistence in this forest raptor guild. Consistent with this idea, the analysis of specific 
nesting locations chosen by sparrowhawks suggest that they make efforts to hide their nests. Sparrowhawks in our study area frequently nested in tree stands with higher local tree density, hidden nests in lower tree strata, and selected evergreen closed-canopy tree species such as Australian blackwood (unpubl.), using mechanisms of concealment already described by other authors (Newton 1986, Selås 1996, 1997, Abe et al. 2007). Our finding that a relatively large proportion of sparrowhawks (94\%) built a new nest every year can also be interpreted as an anti-predation measure to prevent the nest from serving as a fixed spatial reference for the dominant predator (Newton 1986). In addition to this concealment effect, we suggest that buzzards indirectly protected sparrowhawks from predation by goshawks, facilitating nesting by subordinate predators in favorable habitats that they otherwise could not access because of high risk of predation.

5) Diet segregation. Intraguild predation (IGP) implies competition between IG predator (goshawk, the dominant predator) and IG prey (sparrowhawk, the subordinate predator). Low overlap between diets of sparrowhawks and goshawks during the breeding season favors survival of the subordinate predator because it reduces the intensity of the competition. Sparrowhawk diet comprises mainly small birds lighter than $50 \mathrm{~g}$ (Mañosa and Oro 1991, Selås 1993), which make up less than $2 \%$ of goshawk diet in the study area during the breeding season (Garcia-Salgado et al. 2015). Theoretical considerations of intraguild predation (Polis et al. 1989, Polis and Holt 1992, Holt and Polis 1997) predict the coexistence of the IG prey (sparrowhawk) with a high density of IG predator (goshawk) when three circumstances coincide. a) The IG prey is better at capturing its own small prey than the IG predator. b) The IG prey is not a main prey of the IG predator. c) The IG prey can seek protection in spatial refuges. All three conditions were satisfied in our study area.

The results of this study support our initial hypothesis that interspecific interactions within the raptor guild influence the spatial distribution of predator species in forest ecosystems, and also that intraguild predation is a key driver. The spatial relationships detected in the present study were different from those found in a preliminary study where we did not take into account habitat preferences and intraspecific territoriality of species to elaborate the null hypotheses (Rebollo et al. 2011). In that study we detected shorter distances than those expected by chance between sparrowhawks and goshawks, and between goshawks and buzzards, mainly as a consequence of the strong preference of the three species for eucalyptus stands with the oldest and tallest eucalyptus trees and with a diverse tree composition. The different results found in both approaches emphasize the importance of taking into account intraspecific territoriality (second-order factors) and habitat preferences of each species (first-order factors) when analyzing third-order factors (interspecific interactions). It is also interesting to note that we did not detect any significant attraction or repulsion regarding the second and third interspecific neighbor nests. The major role of distances to the first neighbor (NNDs) could be due to the fact that raptor populations are rarely saturated.
We recommend future researches to confirm the potential effects of intraguild interactions on the spatial distribution of predation pressures on prey. Indeed, spatial patterns of aggregation and spacing that we documented may affect the spatial distribution of predation pressures on prey of these central-place foraging raptors (Byholm et al. 2012). On one hand, some subordinate predators nested far from dominant predators, probably generating enemy-free areas around the nests of the dominant species for exclusive prey of the subordinate predators (Thomson et al. 2006, Mönkkönen et al. 2007). On the other hand, non-dominant predator species tended to nest close together, but this appears not to generate areas of more intense predation pressure on shared prey, because the diets of these predators were almost completely different.

Acknowledgements - We are indebted to Enrique de la Montaña, Miguel Rebollo, Pablo Rebollo, Alberto Navarro, Alfredo Cofiño, Ángel Sanz, Myriam Ramírez, Jorge Meltzer, Mar Zurita and Sofía Morcelle for their great help with laboratory and field work, and to Carlos Sobreira for his assistance with prey identification from photographs. We thank Fabio Suzart and Jaime Madrigal for statistical advice. We are grateful to Armando Chapin Rodríguez, Mónica Díaz-Otero and Zoë Rohrer for valuable comments on the manuscript. We acknowledge the Dirección Xeral de Conservación da Natureza of the Xunta de Galicia for granting permission to carry out this study.

Funding - This work was supported by funds from CICYT projects of the Spanish Ministerio de Educación y Ciencia (CGL200760533/BOS, CGL2010-18312/BOS) and Ministerio de Economía y Competitividad (CGL2014-533308-P), projects of the Alcalá Univ. (CCG2014/BIO-002), and the REMEDINAL network (S-0505/AMB/0335, S-2009/AMB/1783 and S-2013/MAE/2719). SMH and GGS were supported by FPI and FPU fellowships from the Ministerio de Educación y Ciencia (BES-2008-006630 and AP2006-00891, respectively).

Author contributions - Conceived and designed the study: SR, SMH, LPC, GGS. Conduct field work: JMFP, SR, SMH, GGS, LPC. Analyzed the data: JJ, SMH, GGS, LPC, SR. Wrote the paper: SR, SMH, GGS, LPC, JMFP, JJ.

Conflicts of interest - The authors declare that they have no conflict of interest.

Permits - All the work was conducted in accordance with relevant national and international guidelines and conforms to the legal requirements of the regional government (Dirección Xeral de Conservación da Natureza of the Xunta de Galicia) which granted permission to carry out the study.

\section{References}

Abe, F., Hasegawa, O., Kudo, T. and Higashi, S. 2007. Nest-site selection of northern goshawks and Eurasian sparrowhawks in a fragmented landscape in northern Japan. - J. Raptor Res. 41: 299-306.

Álvarez-Taboada, M. F. 2005. Remote sensing and Geoinformation Systems applied to the forest management of Eucalyptus globulus Labill. stands damaged by Gonipterus scutellatus Gyllenhal in Galicia. - PhD thesis, Univ. of Vigo, Spain.

Bates, D., Maechler, M., Bolker, B. and Walker, S. 2013. lme4: linear mixed-effects models using $\mathrm{S} 4$ classes. - $\mathrm{R}$ package ver. $1.0-5$.

Björklund, H., Santangeli, A., Guillaume-Blanchet, F., Huitu, O., Lehtoranta, H., Lindén, H., Valkama, J. and Laaksonen, T. 
2016. Intraguild predation and competition impacts on a subordinate predator. - Oecologia 181: 257-269.

Byholm, P., Burgas, D., Virtanen, T. and Valkama, J. 2012. Competitive exclusion within the predator community influences the distribution of a threatened prey species. - Ecology 93: 1802-1808.

Carballeira, A., Devesa, C., Retuerto, R., Santillán, E. and Ucieda, F. 1983. Bioclimatología de Galicia. - Fundación Pedro Barrié de la Maza, Conde de Fenosa, La Coruña, Spain.

Chakarov, N. and Krüger, O. 2010. Mesopredator release by an emergent superpredator: a natural experiment of predation in a three level guild. - PLoS One 5: e15229.

Cornulier, T. and Bretagnolle, V. 2006. Assessing the influence of environmental heterogeneity on bird spacing patterns: a case study with two raptors. - Ecography 29: 240-250.

Cramp, S. and Simmons, K. E. L. 2004. BWPi: birds of the Western Palearctic interactive (DVD-ROM). - BirdGuides, Sheffield.

Del Hoyo, J., Elliot, A. and Sargatal, J. (eds) 1994. Handbook of the birds of the World. Vol. 2. New World vultures to guineafowl. - Lynx Edicions.

ESRI 2010. ArcGIS desktop: release 10. - Environmental Systems Research Inst., Redlands, CA.

Fasola, M. and Zanghellini, S. 1993. Breeding habitats of sparrowhawks (Accipiter nisus) and goshawks (A. gentilis) in the southern Alps. - Avocetta 17: 11-14.

Ferguson-Lees, J. and Christie, D. A. 2001. Raptors of the World. - Christopher Helm.

Gamauf, A., Tebb, G. and Nemeth, E. 2013. Honey buzzard Pernis apivorus nest-site selection in relation to habitat and the distribution of goshawks Accipiter gentilis. - Ibis 155: 258-270.

García-Salgado, G., Rebollo, S., Pérez-Camacho, L., MartínezHesterkamp, S., Navarro, A. and Fernández-Pereira, J. M. 2015. Evaluation of trail-cameras for analyzing the diet of nesting raptors using the northern goshawk as a model. - PLoS One 10: e0127585.

Hakkarainen, H., Mykra, S., Kurki, S., Tornberg, R. and Jungell, S. 2004. Competitive interactions among raptors in boreal forests. - Oecologia 141: 420-424.

Holt, R. D. and Polis, G. A. 1997. A theoretical framework for intraguild predation. - Am. Nat. 149: 745-764.

Katzner, T. E., Bragin, E. A., Knick, S. T. and Smith, A. T. 2003. Coexistence in a multispecies assemblage of eagles in central Asia. - Condor 105: 538-551.

Kenward, R. 2006. The goshawk. - T and AD Poyser.

Kostrzewa, A. 1991. Interspecific interference competition in three European raptor species. - Ethol. Ecol. Evol. 3: 127-143.

Krüger, O. 2002a. Analysis of nest occupancy and nest reproduction in two sympatric raptors: common buzzard Buteo buteo and goshawk Accipiter gentilis. - Ecography 25: 523-532.

Krüger, O. 2002b. Interactions between common buzzard Buteo buteo and goshawk Accipiter gentilis: trade-offs revealed by a field experiment. - Oikos 96: 441-452.

Lourenço, R., Santos, S. M., Rabaça, J. E. and Penteriani, V. 2011. Superpredation patterns in four large European raptors. - Popul. Ecol. 53: 175-185.

Mañosa, S. and Oro, D. 1991. Diet composition during the breeding season of a population of sparrowhawks (Accipiter nisus) in central Catalonia (NE Spain). - Ardeola 38: 289-296.

Mönkkönen, M., Husby, M., Tornberg, R., Helle, P. and Thomson, R. L. 2007. Predation as a landscape effect: the trading off by prey species between predation risks and protection benefits. - J. Anim. Ecol. 76: 619-629.

Morosinotto, C., Villers, A., Thompson, R. L., Varjonen, R. and Korpimäki, E. 2017. Competitors and predators alter settlement patterns and reproductive success of an intraguild prey. - Ecol. Monogr. 87: 4-20.
Mueller, A. K., Chakarov, N., Heseker, H. and Krüger, O. 2016. Intraguild predation leads to cascading effects on habitat choice, behaviour and reproductive performance. - J. Anim. Ecol. 85: 774-784.

Newton, I. 1979. Population ecology of raptors. - T and AD Poyser.

Newton, I. 1986. The sparrowhawk. - T and AD Poyser.

Newton, I., Marquiss, M., Weir, D. N. and Moss, D. 1977. Spacing of sparrowhawk nesting territories. - J. Anim. Ecol. 46: 425-441.

Penteriani, V. and Faivre, B. 1997. Breeding density and landscapelevel habitat selection of common buzzards (Buteo buteo) in a mountain area (Abruzzo Apennines, Italy). - J. Raptor Res. 31: 208-212.

Pérez-Camacho, L., García-Salgado, G., Rebollo, S., MartínezHesterkamp, S. and Fernández-Pereira, J. M. 2015. Higher reproductive success of small males and greater recruitment of large females may explain the strong reversed sexual dimorphism (RSD) in the northern goshawk. - Oecologia 177: 379-387.

Polis, G. A. and Holt, R. D. 1992. Intraguild predation: the dynamics of complex trophic interactions. - Trends Ecol. Evol. 7: 151-154.

Polis, G. A., Myers, C. A. and Holt, R. D. 1989. The ecology and evolution of intraguild predation: potential competitors that eat each other. - Annu. Rev. Ecol. Syst. 20: 297-330.

Rebollo, S., Pérez-Camacho, L., García-Salgado, G., MartínezHesterkamp, S., Fernández-Pereira, J. M., Rebollo, M., Rebollo, P. and de la Montaña, E. 2011. Spatial relationship among northern goshkawk, Eurasian sparrowkawk and common buzzard: rivals or partners? - In: Zuberogoitia, I. and Martínez. J. E. (eds), Ecology and conservation of European forest raptors. Diputación Foral de Bizkaia, Pais Vasco, Spain, pp. 159-167.

Reich, R. M., Joy, S. M. and Reynolds, R. T. 2004. Predicting the location of northern goshawk nests: modeling the spatial dependency between nest locations and forest structure. - Ecol. Model. 176: 109-133.

Reynolds, R. T. and Meslow, E. C. 1984. Partitioning of food and niche characteristics of coexisting Accipiter during breeding. - Auk 101: 761-779.

Rutz, C., Bijlsma, R. G., Marquiss, M. and Kenward, R. E. 2006. Population limitation in the northern goshawk in Europe: a review with case studies. - Stud. Avian Biol. 31: 158-197.

Selås, V. 1993. Selection of avian prey by breeding sparrowhawks Accipiter nisus in southern Norway: the importance of size and foraging behavior of prey. - Ornis Fenn. 70: 144-154.

Selås, V. 1996. Selection and reuse of nest stands by sparrowhawks Accipiter nisus in relation to natural and manipulated variation in tree density. - J. Avian Biol. 27: 56-62.

Selås, V. 1997. Nest-site selection by four sympatric forest raptors in southern Norway. - J. Raptor Res. 31: 16-25.

Selås, V. and Rafoss, T. 1999. Ranging behaviour and foraging habitats of breeding sparrowhawks Accipiter nisus in a continuous forested area in Norway. - Ibis 141: 269-276.

Sergio, F., Boto, A., Scandolara, C. and Bogliani, G. 2002. Density, nest sites, diet, and productivity of common buzzards (Buteo buteo) in the Italian pre-Alps. - J. Raptor Res. 36: 24-32.

Sergio, F., Marchesi, L. and Pedrini, P. 2003. Spatial refugia and the coexistence of a diurnal raptor with its intraguild owl predator. - J. Anim. Ecol. 72: 232-245.

Sergio, F., Rizzolli, F., Marchesi, L. and Pedrini, P. 2004. The importance of interspecific interactions for breeding-site selection: peregrine falcons seek proximity to raven nests. - Ecography 27: 818-826.

Solonen, T. 1993. Spacing of birds of prey in southern Finland. - Ornis Fenn. 70: 129-143.

StatSoft 2007. Statistica (data analysis software system), version 8.0. - <www.statsoft.com >. 
Symonds, M. E. and Moussalli, A. 2011. A brief guide to model selection, multimodel inference and model averaging in behavioural ecology using Akaike's information criterion. - Behav. Ecol. Sociobiol. 65: 13-21.

Tapia, L., Domínguez, J. and Romeu, M. 2007. Diet of common buzzard (Buteo buteo) in northwestern Spain as assessed by direct observation from blinds - Nova Acta Cientifica Compostelana 16: 145-149.

Thomson, R. L., Forsman, J. T., Sardà-Palomera, F. and Mönkkönen, M. 2006. Fear factor: prey habitat selection and its consequences in a predation risk landscape. - Ecography 29: 507-514.

Supplementary material (Appendix JAV-01337 at <www. avianbiology.org/appendix/jav-01337>). Appendix 1 .
Tubbs, C. R. 1974. The buzzard. - David and Charles, London. Zuberogoitia, I. and Martínez, J. E. 2011. Ecology and conservation of European forest-dwelling raptors. - Depto de Agricultura de la Diputación Foral de Bizkaia, Bilbao, Spain.

Zuberogoitia, I., Martínez, J. E., Martinez, J. A., Zabala, J., Calvo, J. F., Castillo, I., Azkona, A., Iraeta, A. and Hidalgo, S. 2006. Influence of management practices on nest site habitat selection, breeding and diet of the common buzzard Buteo buteo in two different areas of Spain. - Ardeola 53: 83-98. 